\title{
Hot air treatment for surface decontamination of table eggs experimentally infected with Salmonella, Listeria, and Escherichia coli
}

\author{
G. Manfreda • C. Cevoli • A. Lucchi • F. Pasquali • \\ A. Fabbri • A. Franchini
}

Published online: 12 May 2010

(C) Springer Science+Business Media B.V. 2010

\begin{abstract}
Hot-air pasteurization was investigated in the EU-funded project "Reducing Egg Susceptibility to Contaminations in Avian Production in Europe (RESCAPE)" as an innovative treatment for surface bacterial decontamination of table eggs. Possible side effects of the treatment on egg quality traits were also studied. The decontamination power of hot air was evaluated over 1 month on shell eggs that were experimentally inoculated with Salmonella enteritidis, Escherichia coli, or Listeria monocytogenes. The $S$. Enteritidis and L. monocytogenes populations on the surfaces of treated eggs showed a significant reduction compared with untreated eggs. No statistically significant results were obtained comparing E. coli loads on treated and untreated eggs. No detrimental effects on quality traits either immediately after treatment or after 28 days of storage at $20^{\circ} \mathrm{C}$ were recorded.
\end{abstract}

Keywords Hot air · Decontamination · Table eggs · Foodborne pathogens

\section{Abbreviations}

EFSA European Food Safety Authority

\section{Introduction}

By 2012, Council Directive 1999/74/EC, defining minimum standards for the welfare of egglaying hens, will abolish conventional cage systems in favor of enriched cages or floor systems to improve hen welfare. However, these rearing systems could present an increased risk to contamination (higher bacterial eggshell contamination with aerobic bacteria as well as with food pathogens) (EFSA 2005), even if there is not a common scientific agreement on this subject (EFSA 2007). Although any treatment of shell eggs is actually forbidden in Europe, the introduction of efficient measures to eggshell decontamination may be envisaged to reduce any

G. Manfreda $(\bowtie) \cdot$ A. Lucchi $\cdot$ F. Pasquali $\cdot$ A. Franchini

Dipartimento di Scienze degli Alimenti, Alma Mater Studiorum, Università di Bologna, Piazza Goidanich, 60, 47023 Cesena, FC, Italy

e-mail: gerardo.manfreda@unibo.it

C. Cevoli $\cdot$ A. Fabbri

Dipartimento di Economia e Ingegneria Agrarie, Alma Mater Studiorum, Università di Bologna, Viale Fanin, 50, 40127 Bologna, Italy 
food safety risk for consumers. In this regard, in the present study, a hot air pasteurization protocol was created during the activities included in the European Project "RESCAPE," and decontamination was evaluated on the eggshell surfaces of table eggs experimentally infected with Salmonella enteritidis, Escherichia coli, and Listeria monocytogenes. Moreover, the impact of treatment on egg quality traits was evaluated on treated and untreated samples.

\section{Materials and methods}

A prototype for hot air treatment was built based on results obtained by a numerical model of thermal interactions between the air, the shell, and the internal egg content (Fabbri et al. 2009).

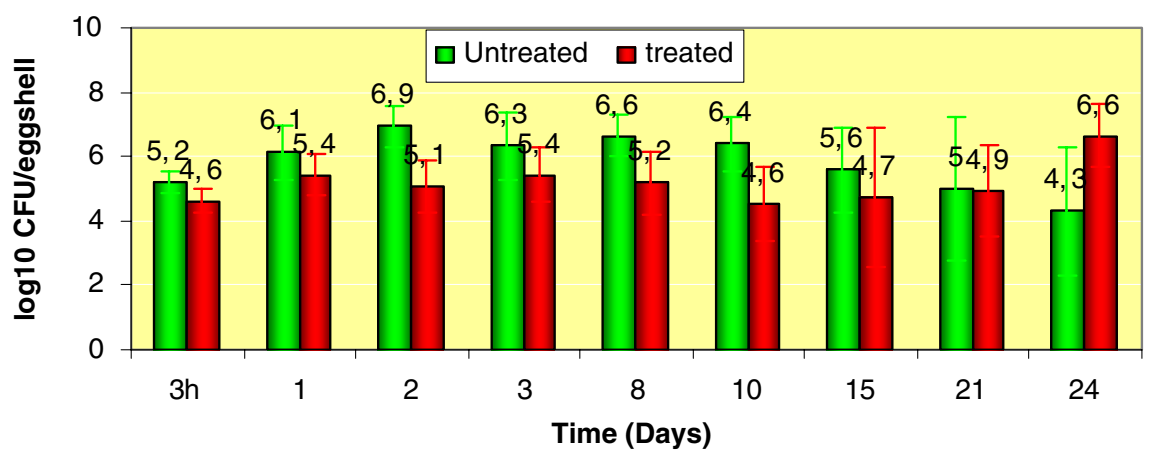

a)

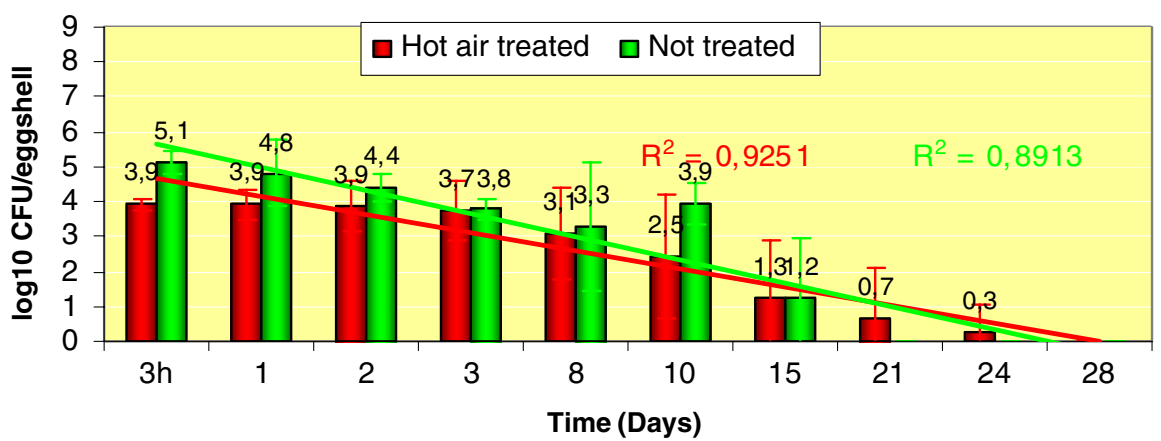

b)

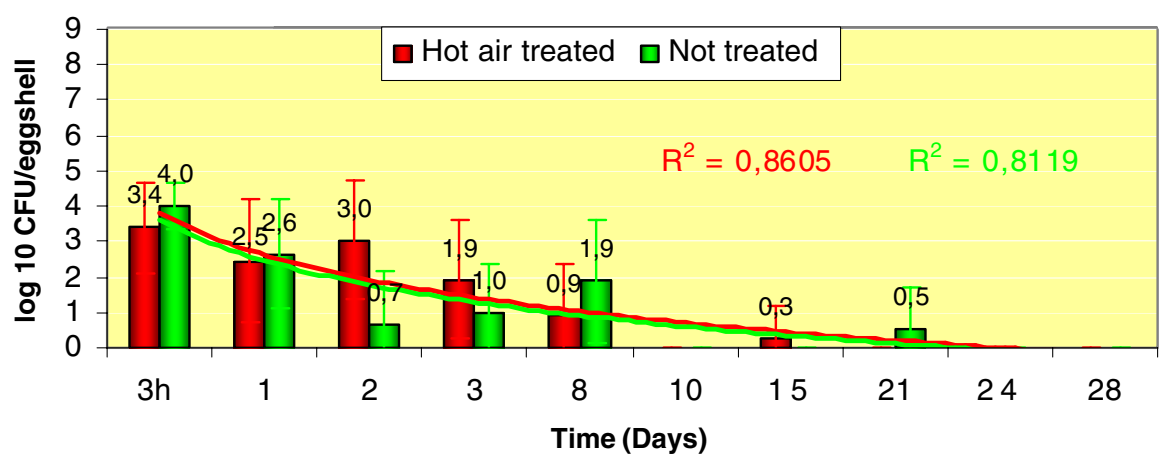

c)

Fig. 1 Comparison among $S$. Enteritidis (a), E. coli (b), and L. monocytogenes (c) loads (log CFU/eggshell) on the shell of untreated and treated eggs 
The best thermal treatment was selected for the highest decontamination efficacy without any negative effects on egg quality. The treatment consisted of two shots of $8 \mathrm{~s}$ each, during which the egg, rotating on rolling cylinders, received hot air at $600^{\circ} \mathrm{C}$ from two hot air generators positioned above the rolling cylinders, and cold air $\left(20-25^{\circ} \mathrm{C}\right)$ from a generator positioned under the rolling cylinders. Between the two shots, the egg received only cold air for $32 \mathrm{~s}$.

The decontamination efficacy of the selected thermal treatment was evaluated for three groups of 200 eggs each that were experimentally contaminated by either S. Enteritidis (MB2509 streptomycin-resistant strain), E. coli (ATCC 25922 strain induced to possess nalidixic acid resistance), or L. monocytogenes (ATCC 13932) using previously described procedures (Hammack et al. 1993). Within each group, 150 shell eggs were treated with hot air and 50 shell eggs were used as controls. After the treatment, all eggs were stored at $20-25^{\circ} \mathrm{C}$ for 28 days. The contamination level on the shell eggs was evaluated at $3 \mathrm{~h}$ and $1,2,3,8,10,15$, 21,24 , and 28 days post-treatment for five untreated and 10 treated eggs per pathogen per day. The bacterial load was determined by surface plate count using the following agar media: Brilliant Green agar (Oxoid, Milan, Italy) supplemented with $25 \mathrm{mg} / \mathrm{L}$ streptomycin for $S$. Enteritidis detection, MacConkey (Oxoid) agar supplemented with $20 \mathrm{mg} / \mathrm{L}$ nalidixic acid for E. coli detection, and Listeria selective agar base (Oxoid) for L. monocytogenes detection.

Different traits were included in the assessment of egg quality. The albumen $\mathrm{pH}$ was measured using a pH meter (CyberScan $510 \mathrm{pH}$, Eutech Instruments) on thick and liquid mixtures of albumen. The turbidity of the albumen, the color of the eggshell, and cuticle assessments were measured as previously described (Weijers et al. 2006; Francis and Clydesdale 1975; Board and Halls 1973). These qualitative traits of 50 treated eggs and 50 untreated eggs were analyzed immediately. The yolk index was calculated as the height of the yolk divided by the width of the yolk measured using a decimal digital calliper. Eggshell breaking strength was measured with a universal testing machine (Egg Shell Force Gauge Model-II, Robotmation Co. Ltd., Tokyo, Japan) on the equatorial area of the egg. These two parameters were evaluated for 50 treated and 50 untreated eggs after 28 days of storage at $20-25^{\circ} \mathrm{C}$.

Table 1 Quality indexes of treated and untreated eggs

\begin{tabular}{lllll}
\hline & Treated eggs & S. D.* & Untreated eggs & S.D.* \\
\hline $\mathrm{pH}$ & 8.96 & 0.2 & 8.97 & 0.30 \\
Turbidity $[/ \mathrm{m}]$ & 41.60 & 7.30 & 43.44 & 7.40 \\
\hline$\Delta E$ (shell colour) & & & 0.65 & \\
$\Delta E$ (cuticle) & & & & \\
\hline Breaking strength (N) & 39.56 & 5.79 & 38.71 & 5.82 \\
Yolk index & 0.17 & 0.03 & 0.16 & 0.02 \\
Weight loss after 7 days (\%) & 1.41 & 16.2 & 1.40 & 20.9 \\
Weight loss after 14 days (\%) & 2.72 & 14.7 & 2.71 & 13.8 \\
Weight loss after 21 days (\%) & 4.91 & 14.4 & 5.16 & 13.2 \\
Weight loss after 28 days (\%) & 6.77 & 10.8 & 6.88 & 12.4 \\
\hline
\end{tabular}

S. D. = standard deviation 


\section{Results}

During the month of storage, the $S$. Enteritidis load reduction on eggshells of treated eggs compared with untreated eggs ranged between 0.1 and 1.9 logs CFU/eggshell (Fig. 1). The E. coli load was heterogeneous during storage (Fig. 1). In particular, a reduction in bacterial load registered only at $0,1,8$, and 21 days post-treatment with values ranging from 0.1 to $0.9 \log _{10} \mathrm{CFU} /$ eggshell without any significant differences. For L. monocytogenes (Fig. 1), the decontamination efficacy was high immediately after treatment with a log reduction of 1.2 logs CFU/eggshell. At the end of the storage time, the loads of both E. coli and $L$. monocytogenes were under the detection limit for both treated and untreated eggs. This decrease was not due to bacterial penetration into the egg, since no contamination was detected in the albumen. A possible explanation may be the low resistance of these bacteria during egg storage. No statistically significant differences were found between the quality traits of treated and untreated eggs (Table 1), indicating no detrimental effects of the hot air treatment.

\section{Discussion}

A mean of $1 \log$ reduction in the $S$. Enteritidis load on treated eggs compared to untreated eggs is an interesting result since it corresponds to a decrease of $90 \%$ of the bacterial population. This decrease may significantly reduce the risk of human salmonellosis if we consider that the Salmonella load on the surfaces of naturally contaminated shell eggs collected from traditional poultry farms rarely exceeds $10^{2} \mathrm{CFU} /$ eggshell (Humphrey et al. 1991). The lack of any effect on quality parameters together with the microbiological results on experimentally inoculated eggs suggest the possible industrial applicability of the hot air decontamination technique for the reduction of foodborne pathogens like $S$. Enteritidis and L. monocytogenes.

Acknowledgments The authors would like to thank W. Messens for kindly providing the $S$. Enteritidis strain. This work was funded by the European Community within the FP6 European project named RESCAPE (Food-CT-2006-036018).

\section{References}

Board RG, Halls NA (1973) The cuticle: A barrier to liquid and particle penetration of the shell of hen's egg. Br Poult Sci 14:67-97

European Food Safety Authority (2005) The welfare aspects of various systems of keeping laying hens. EFSA J 197:1-23

European Food Safety Authority (2007) Report of the Task Force on Zoonoses Data Collection on the Analysis of the baseline study on the prevalence of Salmonella in holdings of laying hen flocks of Gallus gallus. EFSA J 97

Fabbri A, Cevoli C, Giunchi A (2009) Validation of a simplified CFD Model for hot air treatment of shell eggs surface. J Food Process Eng

Francis FJ, Clydesdale FM (1975) Food Colorimetry: Theory and Applications. AVI Publishing, Westport, CT

Hammack TS, Sherrod PS, Bruce VR, June GA, Satchell FB, Andrews WH (1993) Research note: Growth of Salmonella enteritidis in Grade A eggs during prolonged storage. Poult Sci 72:373-377

Humphrey TJ, Whitehead A, Gawler AH, Henley A, Rowe B (1991) Numbers of Salmonella enteritidis in the contents of naturally contaminated hens' eggs. Epidemiol Infect 106:489-496

Weijers M, Van de Velde F, Stijnman A, Van de Pijpekamp A, Visshersa RW (2006) Structure and rheological properties of acid induced egg white protein gels. Food Hydrocolloids 20:146-159 